\title{
A new measurement site for echocardiographic epicardial adipose tissue thickness and its value in predicting metabolic syndrome
}

\author{
Meng Wang ${ }^{1,2, B, D}$, Liang Zhao $0^{1,3, C, D}$, Hao Liang $^{4, B, C}$, Chunyuan Zhang ${ }^{5, B, E}$, Liying Guan ${ }^{6, E}$, Minglong Li Li,A,F \\ ${ }^{1}$ Department of Endocrinology, Shandong Provincial Hospital Affiliated to Shandong University, Jinan, China \\ 2 Department of Endocrinology, Yidu Central Hospital of Weifang, China \\ ${ }^{3}$ Second Department of Endocrinology, Central Hospital of Taian, China \\ ${ }^{4}$ Department of Ultrasonic Diagnosis and Treatment, Shandong Provincial Hospital Affiliated to Shandong University, Jinan, China \\ ${ }^{5}$ Health Management Center, Yidu Central Hospital of Weifang, China \\ ${ }^{6}$ Health Management Center, Shandong Provincial Hospital Affiliated to Shandong University, Jinan, China \\ A - research concept and design; B - collection and/or assembly of data; C - data analysis and interpretation; \\ $D$ - writing the article; $E$ - critical revision of the article; $F$ - final approval of the article
}

Address for correspondence

Minglong Li

E-mail: liminglong_doctors@163.com

Funding sources

None declared

Conflict of interest

None declared

Received on August 11, 2018

Reviewed on December 27, 2018

Accepted on February 18, 2019

Published online on August 30, 2019

Cite as

Wang M, Zhao L, Liang H, Zhang C, Guan L, Li M. A new measurement site for echocardiographic epicardial adipose tissue thickness and its value in predicting metabolic syndrome. Adv Clin Exp Med. 2019;28(10):1403-1408.

doi:10.17219/acem/104526

DOI

$10.17219 /$ acem/104526

Copyright

Copyright by Author(s)

This is an article distributed under the terms of the

Creative Commons Attribution Non-Commercial License

(http://creativecommons.org/licenses/by-nc-nd/4.0/)

\section{Abstract}

Background. Echocardiographic epicardial adipose tissue (EAT) thickness is defined as the thickness of the low-isoechoic area on the free wall of the right ventricle in the parasternal long-axis and short-axis views. Recent studies have suggested that it might support current risk stratification strategies in identifying an increased risk of metabolic syndrome.

Objectives. The aim of this study is to explore a new measurement site which can better reflect EAT thickness and to assess its value in predicting metabolic syndrome.

Material and methods. A total of 975 Chinese adults were measured for EAT thickness on the right ventricular anterior free wall (EAT-rv) and on the anterior interventricular groove (EAT-ivg) with echocardiography. The correlation between EAT thickness and metabolic syndrome was analyzed, as was the agreement between epicardial adipose volume (EAV) and EAT thickness. Independent risk factors of EAT thickness were identified and the predictive value of EAT thickness was assessed.

Results. Epicardial adipose tissue thickness was higher in older participants and those with obesity, diabetes, hypertension, hypertriglyceridemia, and metabolic syndrome, and it was lower in male participants. The EAT-ivg was higher in the participants with hypo-high-density-lipoprotein cholesterolemia than in those without the disorder, but the EAT-rv values were not statistically different. The kappa value was 0.524 between EAT-rv and EAV, and 0.783 between EAT-ivg and EAV. Advanced age, large waist circumference and female gender were independent risk factors of high EAT-ivg, while high-density-lipoprotein (HDL) cholesterol was a protective factor. The EAT-ivg was associated with metabolic syndrome. The area under the curve of EAT-ivg applied in predicting metabolic syndrome was greater than that of EAT-rv (0.715 vs 0.648).

Conclusions. The EAT-ivg was more consistent with EAV than EAT-rv, was independently associated with metabolic syndrome and had a higher value in predicting metabolic syndrome than EAT-rv. Therefore, the anterior interventricular groove can serve as a new measurement site which better reflects EAT thickness.

Key words: metabolic syndrome, echocardiography, epicardial adipose tissue, consistency, predictive value 


\section{Introduction}

Epicardial adipose tissue (EAT) is the visceral thoracic fat depot that surrounds the heart, located between the myocardium and the visceral pericardium. It has been reported that EAT is associated with multiple pathological states, including metabolic syndrome, T2DM, atrial fibrillation, and coronary artery disease. ${ }^{1-3}$ Recent studies have shown that EAT thickness measured with echocardiography may play a limited additional role supporting current risk stratification strategies in identifying individuals at an increased risk of metabolic syndrome. ${ }^{4,5}$ Moreover, measurements of EAT thickness attained by echocardiography and computed tomography (CT) are inconsistent. 6,7 This fact may be explained by the poor distribution of EAT at the measurement site. Echocardiographic EAT thickness has been defined as the thickness of the low-isoechoic area on the free wall of the right ventricle in the parasternal long-axis and shortaxis views, ${ }^{8,9}$ but the distribution of EAT is unbalanced and is concentrated primarily in the interventricular and atrioventricular grooves rather than in the free wall of the right ventricle. ${ }^{7}$ Therefore, there is clinical value in finding a new measurement site for echocardiographic EAT thickness which can better reflect the risk of metabolic syndrome. In addition, Salami et al. have confirmed that there are significant racial differences in the distribution of EAT. ${ }^{10}$

A large-sample investigation of EAT thickness in Chinese adults has not yet been published. In this study, a total of 975 Chinese adults were measured for EAT thickness on the right ventricular anterior free wall (EAT-rv) and on the anterior interventricular groove (EAT-ivg). Distribution of EAT and its consistency with epicardial adipose volume (EAV) were described, and its independent association with metabolic syndrome and its value in predicting metabolic syndrome were analyzed. The aims were to explore a new measurement site which can better reflect EAT thickness and to assess its value in predicting metabolic syndrome.

\section{Material and methods}

\section{Participants}

A total of 986 Chinese adults receiving a general check-up at the Health Management Center of Shandong Provincial Hospital between January 2017 and September 2017 were enrolled in the study. The exclusion criteria consisted of the following: 1 ) age $<18$ years or $>75$ years; 2 ) atrial fibrillation; 3) serum creatinine level $>2 \mathrm{mg} / \mathrm{dL} ; 4$ ) hypothyroidism; 5) current or past use of glucagon-like peptide (GLP)-1, SGLT2 inhibitors, thiazolidinediones, fibrates, statins, or insulins; 6) coronary heart disease; and 7) advanced malignant tumors. Transthoracic 2-dimensional echocardiography was performed in all participants, and 11 participants were excluded because of inadequate image quality. This study was approved by the ethics committee of Shandong
Provincial Hospital, China (approval No. 2016024138), and all participants provided their written informed consent.

\section{Measurement of EAT thickness}

Transthoracic 2-dimensional echocardiography was performed with a Philips iU22 ultrasound system and an 55-1 matrix-array transducer (Phillips Healthcare, Bothell, USA) according to the recommendations of the European and American Societies of Echocardiography. ${ }^{11,12}$ All participants were placed in the left lateral decubitus position. Ultrasonic images were stored in specialized workstations where EAT thickness was measured by the same reader. According to recommendations by Iacobellis et al., EAT thickness on the right ventricular anterior free wall (EAT-rv) was measured, ${ }^{8,9}$ as shown in Fig. 1. In addition, EAT thickness on the anterior interventricular groove (EAT-ivg) at the midchordal level and the tip of the papillary muscle from the parasternal short-axis view was measured as a new measurement site, as shown in Fig. 2. The average value of 3 cardiac cycles for each echocardiographic view was used for analysis.

\section{Quantification of epicardial adipose volume}

Among these 975 participants, 120 were randomly selected for cardiac dual-source computer tomography (DSCT). Cardiac DSCT was performed using a 128-slice DSCT scanner (Siemens AG, Munich, Germany). Image reconstructions were performed at 75\% RR intervals. A density ranging from -30 to -190 Hounsfield units was employed to identify adipose tissue. The pericardium was manually traced from the right pulmonary artery to the diaphragm in order to determine a region of interest. Epicardial adipose volume was assessed using a dedicated workstation (Syngo.via v. VB10A; Siemens) by the same experienced CT diagnostic physician.

\section{Diagnosis of metabolic syndrome}

Metabolic syndrome was determined according to the recommendations of the Chinese Diabetes Society, ${ }^{13}$ including: 1) obesity, i.e., body mass index (BMI) $\geq 25 \mathrm{~kg} / \mathrm{m}^{2}$; 2) fasting blood glucose level $\geq 6.1 \mathrm{mmol} / \mathrm{L}$ or the use of antidiabetic medication; 3) triglyceride level $\geq 1.7 \mathrm{mmol} / \mathrm{L}$; 4) high-density-lipoprotein (HDL) cholesterol count $<1.04 \mathrm{mmol} / \mathrm{L}$; and 5) blood pressure higher than 130/85 $\mathrm{mm} \mathrm{Hg}$ or the use of hypotension medication. Metabolic syndrome was diagnosed when a patient had at least 3 of the above 5 components.

\section{Statistical analysis}

All analyses were performed using SPSS software v. 22.0 (IBM Corp., Armonk, USA). Continuous variables with 


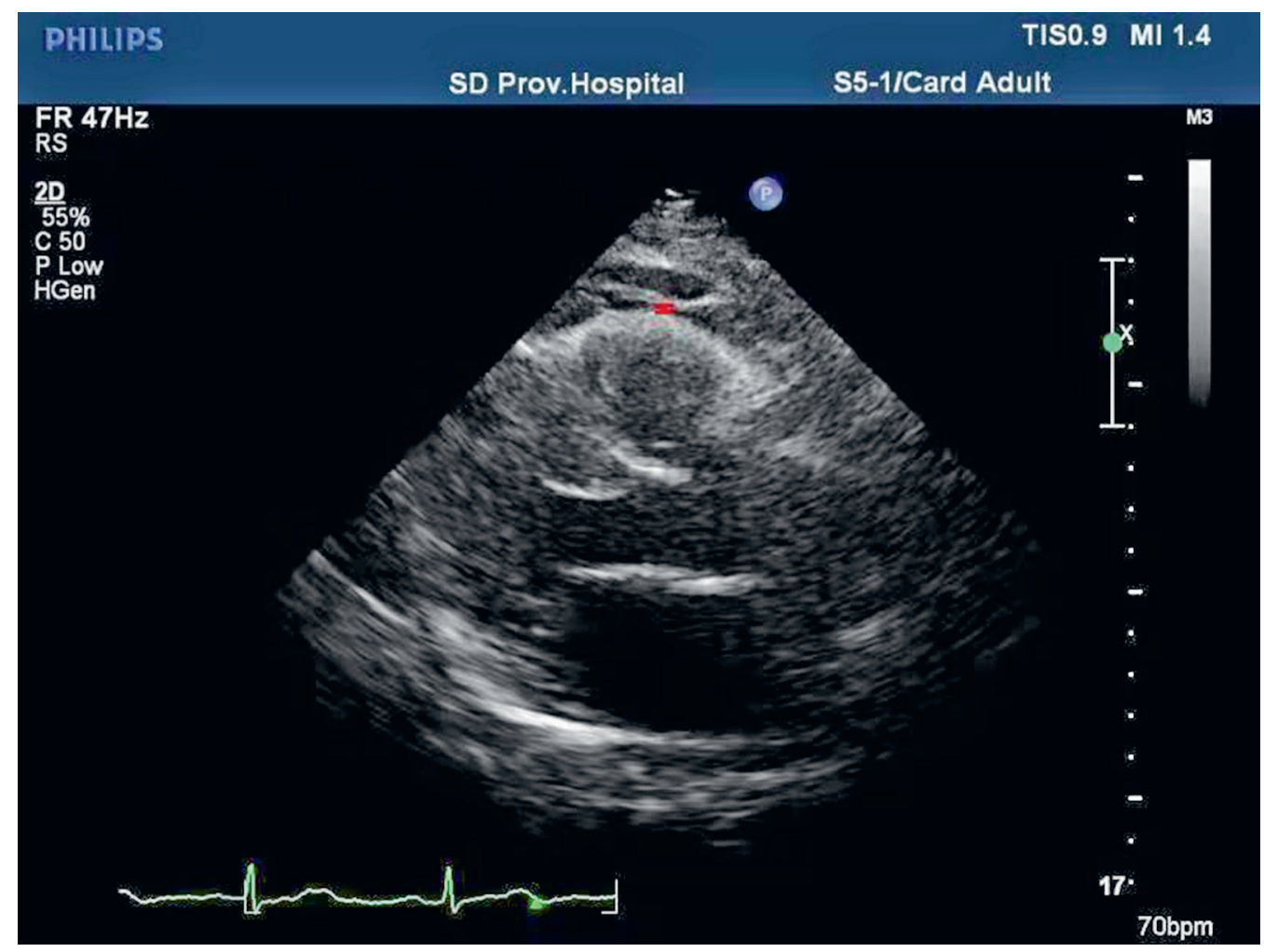

Fig. 1. Measurement of EAT-rv on the right ventricular anterior free wall during end-systole, according to recommendations by lacobellis et al. The red

line shows the measurement distance

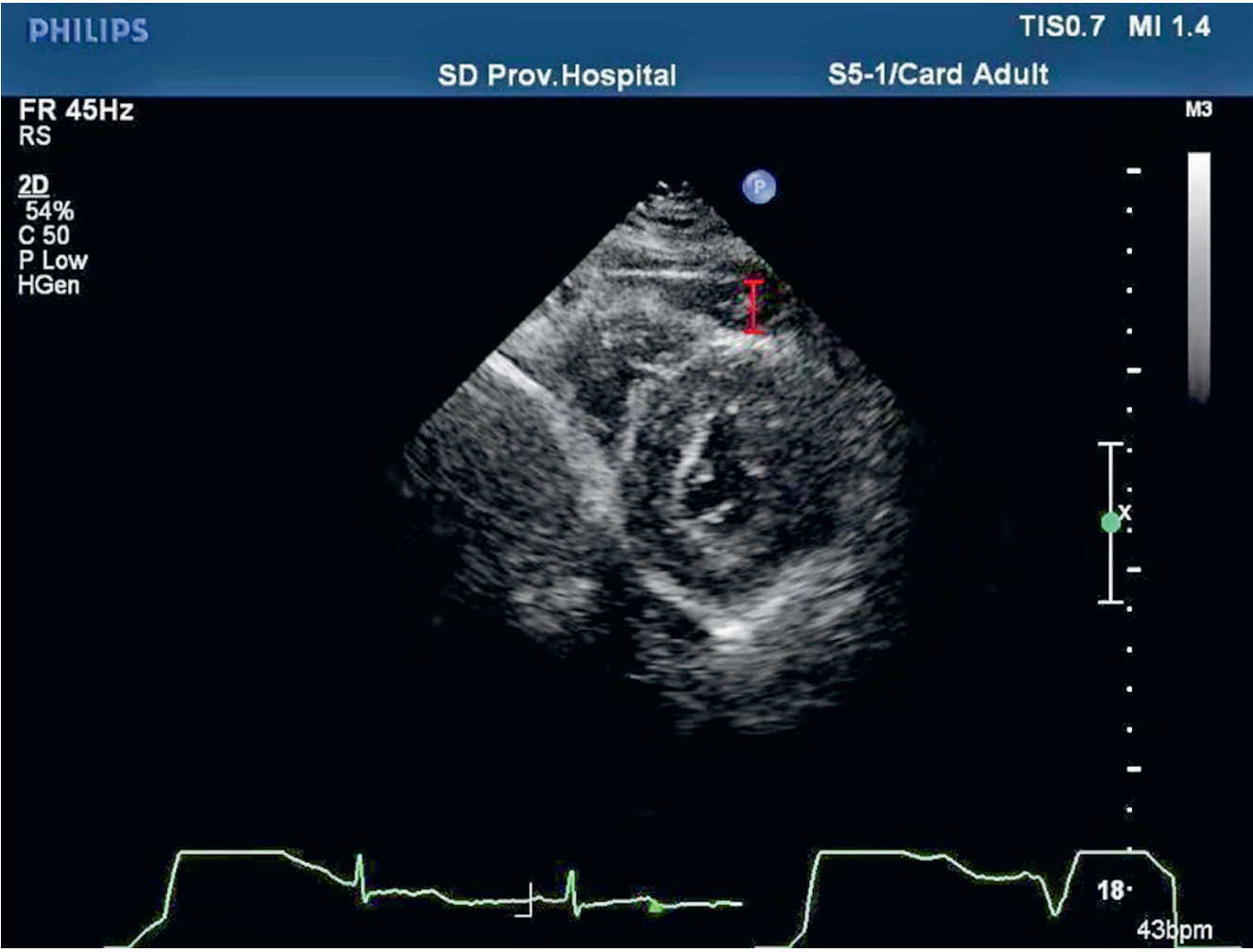

Fig. 2. Measurement of EAT-ivg on the anterior interventricular groove in the parasternal shortaxis view. The red line shows the measurement distance

normal distribution were expressed as means \pm standard deviations (SDs) and those without normal distribution as $25^{\text {th }}-75^{\text {th }}$ percentiles (P25-P75). The EAT thicknesses were compared between groups using the Kruskal-Wallis test. Non-conditional logistic regression analysis was performed to determine the association between metabolic syndrome and EAT thickness. The EAV and EAT thickness were grouped according to tertiles, and consistency was determined with $\mathrm{k}$ statistics. Ordered logistic regression was used to identify independent risk factors of EAT thickness (quartiles). The predictive value of EAT thickness was evaluated with a receiver operating characteristic (ROC) curve, and the areas under the curve (AUC) were compared with the Z-test. Significance was set at $\mathrm{p}<0.05$. 


\section{Results}

\section{Distribution of EAT thickness}

The mean age of all participants $(\mathrm{n}=975)$ was 55.58 \pm 11.78 years; their EAT-rv was $3.01(2.09-3.90)$ and their EAT-ivg was 5.27 (4.01-6.86). EAT-ivg was greater than EAT-rv $(p<0.05)$. The distribution of EAT thickness in all participants is shown in Table 1. Epicardial adipose tissue thickness was higher in older participants and in those with obesity, diabetes, hypertension, hypertriglyceridemia, and metabolic syndrome; it was lower among the male participants. However, EAT-ivg was higher in participants with hypo-high-density-lipoprotein cholesterolemia than in those without this condition, and EAT-rv was not statistically different between the participants with and without hypo-high-density-lipoprotein cholesterolemia.

Table 1. Distribution of EAT thickness in all participants (data expressed as median (P25-P75))

\begin{tabular}{|c|c|c|c|}
\hline Parameter & $\mathrm{n}$ & EAT-rv [mm] & EAT-ivg [mm] \\
\hline \multicolumn{4}{|c|}{ Sex } \\
\hline male & 431 & $2.66(2.02-3.65)$ & $5.19(3.70-6.63)$ \\
\hline female & 544 & $3.17(2.18-4.05)^{*}$ & $5.38(4.06-7.21)^{\&}$ \\
\hline \multicolumn{4}{|c|}{ Age [years] } \\
\hline$\leq 45$ & 211 & $2.03(1.47-2.91)$ & $3.93(2.65-5.00)$ \\
\hline $46-55$ & 248 & $2.63(2.03-3.48)$ & $5.04(3.74-6.13)$ \\
\hline $56-65$ & 282 & $3.22(2.36-3.95)$ & $6.07(4.54-7.26)$ \\
\hline $66-75$ & 234 & $3.73(3.02-4.55)^{*}$ & $6.49(5.05-8.28)^{*}$ \\
\hline \multicolumn{4}{|c|}{ BMI $\left[\mathrm{kg} / \mathrm{m}^{2}\right]$} \\
\hline$\leq 23.9$ & 375 & $2.78(2.02-3.73)$ & $4.72(3.28-6.27)$ \\
\hline $24.0-27.9$ & 434 & $3.02(2.10-3.90)$ & $5.36(4.08-7.00)$ \\
\hline$\geq 28$ & 165 & $3.41(2.32-4.35)^{*}$ & $6.41(4.88-7.92)^{*}$ \\
\hline \multicolumn{4}{|c|}{ Diabetes } \\
\hline yes & 169 & $3.39(2.32-4.18)^{*}$ & $6.35(4.29-8.20)^{*}$ \\
\hline no & 805 & $2.92(2.04-3.82)$ & $5.09(3.83-6.67)$ \\
\hline \multicolumn{4}{|c|}{ Hypertension } \\
\hline yes & 458 & $3.35(2.52-4.22)^{*}$ & $6.22(4.76-7.58)^{*}$ \\
\hline no & 517 & $2.49(1.92-3.45)$ & $4.23(3.20-6.16)$ \\
\hline \multicolumn{4}{|c|}{ Hypertriglyceridemia } \\
\hline yes & 354 & $3.23(2.30-4.09)^{*}$ & $5.96(4.42-7.30)^{*}$ \\
\hline no & 621 & $2.69(2.02-3.64)$ & $4.90(3.49-6.56)$ \\
\hline \multicolumn{4}{|c|}{ Hypo-high-density-lipoprotein cholesterolemia } \\
\hline yes & 212 & $3.11(2.10-3.90)$ & $5.72(4.35-7.18)^{*}$ \\
\hline no & 763 & $2.91(2.03-3.79)$ & $5.05(3.77-6.82)$ \\
\hline \multicolumn{4}{|c|}{ Metabolic syndrome } \\
\hline yes & 362 & $3.42(2.48-4.24)^{*}$ & $6.44(5.04-7.93)^{*}$ \\
\hline no & 613 & $2.67(2.02-3.63)$ & $4.70(3.29-6.25)$ \\
\hline
\end{tabular}

${ }^{*} p<0.001 ;{ }^{\&} p<0.05$. EAT - epicardial adipose tissue; EAT-rv - EAT thickness on the right ventricular anterior free wall; EAT-ivg - EAT thickness on the anterior interventricular groove; BMI - body mass index.

\section{Consistency between EAT thickness and EAV}

The $\kappa$ value between EAT-rv and EAV was 0.524 $(\mathrm{p}<0.05)$, and that between EAT-ivg and EAV it was 0.783 $(\mathrm{p}<0.05)$ (Table 2 and 3$)$.

Table 2. Agreement analysis by tertiles of EAT-rv and EAV

\begin{tabular}{|l|c|c|c|}
\multirow{2}{*}{ Tertiles of EAV [mL] } & \multicolumn{3}{|c|}{ Tertiles of EAT-rv [mm] } \\
\cline { 2 - 4 } & $<2.32$ & $2.32-3.65$ & $>3.65$ \\
\hline$<45.3$ & $16(13.33 \%)$ & $12(10.00 \%)$ & $12(10.00 \%)$ \\
\hline $45.3-66.2$ & $13(10.83 \%)$ & $17(14.17 \%)$ & $10(8.33 \%)$ \\
\hline$>66.2$ & $11(9.17 \%)$ & $11(9.17 \%)$ & $18(15.00 \%)$ \\
\hline
\end{tabular}

EAT - epicardial adipose tissue; EAT-rv - EAT thickness on the right ventricular anterior free wall; EAV - epicardial adipose volume.

Table 3. Agreement analysis by tertiles of EAT-ivg and EAV

\begin{tabular}{|l|c|c|c|}
\multirow{2}{*}{$\begin{array}{c}\text { Tertiles of EAV } \\
{[\mathrm{mL}]}\end{array}$} & \multicolumn{3}{|c|}{ Tertiles of EAT-ivg [mm] } \\
\cline { 2 - 4 } & $<4.43$ & $4.43-6.67$ & $>6.67$ \\
\hline$<45.3$ & $19(15.83 \%)$ & $11(9.17 \%)$ & $10(8.33 \%)$ \\
\hline $45.3-66.2$ & $12(10.00 \%)$ & $18(15.00 \%)$ & $10(8.33 \%)$ \\
\hline$>66.2$ & $9(7.50 \%)$ & $11(9.17 \%)$ & $20(16.67 \%)$ \\
\hline
\end{tabular}

EAT - epicardial adipose tissue; EAT-ivg - EAT thickness on the anterior interventricular groove; EAV - epicardial adipose volume.

\section{Risk factors of EAT-ivg}

As shown in Table 4, ordered logistic regression analysis showed that advanced age, large waist circumference and female gender were independent risk factors of high EAT-ivg, and HDL cholesterol was a protective factor. On the other hand, BMI, triglyceride, HDL cholesterol, uric acid, creatinine, estimated glomerular filtration rate (eGFR), and Hba1c levels, hypertension, diabetes, and lifestyle (including eating habits, smoking and exercise habit) were not statistically significant.

Table 4. Risk factors of high EAT-ivg

\begin{tabular}{|l|c|c|c|}
\multicolumn{1}{|c|}{ Parameter } & $\begin{array}{c}\text { Wald } \\
\text { statistic }\end{array}$ & ORs $(95 \% \mathrm{Cl})$ & p-value \\
\hline Age [years] & 29.977 & $1.061(1.037-1.086)$ & $<0.001$ \\
\hline HDL cholesterol $[\mathrm{mmol} / \mathrm{L}]$ & 6.379 & $0.431(0.225-0.825)$ & $<0.05$ \\
\hline Waist circumference $[\mathrm{cm}]$ & 11.283 & $1.116(1.048-1.187)$ & $<0.05$ \\
\hline Female gender & 13.642 & $2.674(1.559-4.587)$ & $<0.001$ \\
\hline
\end{tabular}

OR - odds ratio; 95\% Cl - 95\% confidence interval; HDL cholesterol - highdensity-lipoprotein cholesterol.

\section{Association between EAT thickness and metabolic syndrome}

As shown in Table 5, non-conditional logistic regression analysis revealed that EAT-ivg was associated with metabolic syndrome, and the crude, age-adjusted, 
Table 5. Association between EAT metrics and metabolic syndrome

\begin{tabular}{|l|c|c|c|c|}
\multicolumn{1}{|c|}{ Parameter } & EAT-rv & p-value & EAT-ivg \\
\hline Crude OR & $1.337(1.162-1.538)$ & $<0.001$ & $1.628(1.498-1.770)$ \\
\hline Age-adjusted OR & $1.306(1.124-1.518)$ & $<0.001$ & $1.569(1.457-1.690)$ \\
Age- and waist-circumference-adjusted OR & $1.163(0.985-1.374)$ & 0.075 & $1.363(1.221-1.520)$ \\
\hline Age-, waist-circumference- and BMl-adjusted OR & $1.116(0.938-1.329)$ & 0.217 & $<0.001$ \\
\hline
\end{tabular}

EAT - epicardial adipose tissue; EAT-rv - EAT thickness on the right ventricular anterior free wall; EAT-ivg - EAT thickness on the anterior interventricular groove; BMI - body mass index; OR - odds ratio.

age- and waist-circumference-adjusted and age-, waistcircumference- and BMI-adjusted odds ratios (ORs) were $1.628,1.529,1.363$, and 1.331, respectively. The EAT-rv was associated with metabolic syndrome when only age was adjusted for.

\section{Value of EAT thickness applied in predicting metabolic syndrome}

As shown in Fig. 3, the AUC of EAT-rv applied in predicting metabolic syndrome was 0.648 (standard error: 0.018 ), and the AUC of EAT-ivg was 0.715 (standard error: 0.017). A Z-test showed that the AUC of EAT-ivg was greater than that of EAT-rv $(\mathrm{p}<0.05)$.

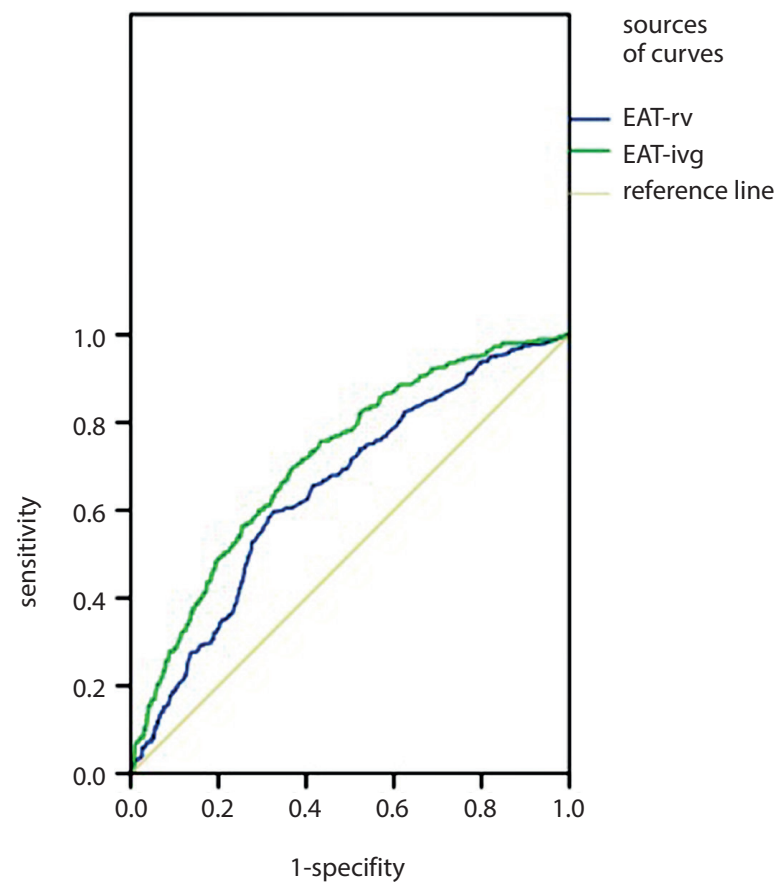

Fig. 3. The AUC of EAT-rv and EAT-ivg applied in predicting metabolic syndrome

\section{Discussion}

To our knowledge, this is the first large-scale clinical study to investigate the distribution of EAT and its association with metabolic syndrome. In this study, we explored a new measurement site for echocardiographic
EAT thickness (EAT-ivg), which was greater than EAT-rv. The median values for EAT-ivg and EAT-rv were 5.27 (4.01-6.86) and 3.01 (2.09-3.90), respectively. The EAT-rv values found among our sample of the Chinese population were similar to those of South Koreans, ${ }^{14}$ but lower than values measured among Caucasians. ${ }^{15}$ This racial difference was also confirmed by Fox et al. ${ }^{16}$ Our results show that EAT thickness was higher in older people and lower in males, a finding which is consistent with a study by Graeff et al. ${ }^{4}$ Both clinical studies and autopsies confirmed that EAT thickness significantly correlates with aging. ${ }^{15,17}$ As for gender difference, our results were consistent with the study by Baragetti et al., ${ }^{18}$ but opposite to the study by Calabuig et al. ${ }^{15}$ This discrepancy could be explained by the fact that the latter study did not include multivariate analysis.

In 2003, Iacobellis et al. first measured EAT thickness on the right ventricle anterior free wall (EAT-rv) with echocardiography, which showed an excellent agreement with MRI epicardial measurements and a significant association with metabolic syndrome. ${ }^{8}$ However, the association was reduced when the size of the study population was increased and multivariate analysis was performed. ${ }^{4,15}$ In our study, EAT-rv also lacked a significant correlation with metabolic syndrome after multivariate analysis, but EAT-ivg did significantly correlate with metabolic syndrome. In addition, the $\mathrm{k}$ value between EAT-rv and EAV was lower than that of EAT-ivg and EAV, and the AUC for the application of EAT-rv in predicting metabolic syndrome was lower than that of EAT-ivg. Therefore, EAT-ivg is a better index for echocardiographic EAT thickness and is more valuable in predicting metabolic syndrome.

We also analyzed the independent risk factors of EAT thickness. In addition to age and sex, waist circumference - a commonly used assessment tool for visceral fat - was also an independent risk factor. This conclusion is consistent with previous reports. ${ }^{4,5,15}$ High-densitylipoprotein cholesterol is a protective factor of EAT thickness, according to our results. In a cross-sectional study of 72 hemodialysis patients, a low HDL cholesterol level was identified as an independent risk factor of elevated EAT thickness. ${ }^{19}$ A meta-analysis showed that there was a highly significant $(\mathrm{p}<0.001)$ correlation between EAT thickness and HDL cholesterol. ${ }^{5}$ Our results confirm the previous findings. 
One advantage of this study was employing an echocardiogram to measure EAT thickness in different locations, and then to compare them with EAV as measured with CT. The distribution of EAT thickness was asymmetrical, and the EAT on the anterior interventricular groove was thicker than on the right ventricle anterior free wall. We chose the anterior interventricular groove as the anatomic landmark in the parasternal short-axis view because this landmark was the most definitive and had the maximum distribution of EAT we could find using transthoracic echocardiography. We also compared EAT-rv and EAT-ivg with EAV. Their $\kappa$ coefficients were small, which was similar to a study by Kim et al., ${ }^{20}$ but EAT-ivg had a larger $\mathrm{k}$ coefficient than EAT-rv. This meant that EAT-ivg correlated more strongly with EAV than EAT-rv.

There were also some limitations in our study. Firstly, the participants were people undergoing physical examination, which may have led to selection bias. Secondly, cardiac magnetic resonance is considered the gold standard and it can more accurately assess epicardial fat than transthoracic echocardiography and cardiac DSCT. However, it is expensive and difficult to perform in clinical practice for a large-scale cohort. Thirdly, we did not measure visceral fat because it is also difficult to measure in a large population.

\section{Conclusions}

In summary, EAT-ivg had a stronger agreement with EAV than EAT-rv, it was independently associated with metabolic syndrome and it was more valuable in predicting metabolic syndrome than EAT-rv. Therefore, the anterior interventricular groove can serve as a new measurement site which can better reflect EAT thickness.

\section{ORCID iDs}

Meng Wang (D) https://orcid.org/0000-0002-7663-6756 Liang Zhao (1) https://orcid.org/0000-0003-1230-2199 Hao Liang (1) https://orcid.org/0000-0002-8902-6199 Chunyuan Zhang (1) https://orcid.org/0000-0001-7832-7714 Liying Guan (1) https://orcid.org/0000-0001-9648-6698 Minglong Li (i) https://orcid.org/0000-0003-3870-5519

\section{References}

1. Al Chekakie MO, Welles CC, Metoyer R, et al. Pericardial fat is independently associated with human atrial fibrillation. J Am Coll Cardiol. 2010;56(10):784-788.

2. Clément $K$, Basdevant $A$, Dutour $A$. Weight of pericardial fat on coronaropathy. Arterioscler Thromb Vasc Biol. 2009;29(5):615-616.
3. Ouwens DM, Sell H, Greulich S, Eckel J. The role of epicardial and perivascular adipose tissue in the pathophysiology of cardiovascular disease. J Cell Mol Med. 2010;14(9):2223-2234.

4. Graeff DB, Foppa M, Pires JC, et al. Epicardial fat thickness: Distribution and association with diabetes mellitus, hypertension and the metabolic syndrome in the ELSA-Brasil study. Int J Cardiovasc Imaging. 2016;32(4):563-572.

5. Rabkin SW. The relationship between epicardial fat and indices of obesity and the metabolic syndrome: A systematic review and metaanalysis. Metab Syndr Relat Disord. 2014;12(1):31-42.

6. Kim BJ, Kim HS, Kang JG, Kim BS, Kang JH. Association of epicardial fat volume and nonalcoholic fatty liver disease with metabolic syndrome: From the CAESAR study. J Clin Lipidol. 2016;10(6):1423-1430.e1.

7. Wang TD, Lee WJ, Shih FY, et al. Relations of epicardial adipose tissue measured by multidetector computed tomography to components of the metabolic syndrome are region-specific and independent of anthropometric indexes and intraabdominal visceral fat. J Clin Endocrinol Metab. 2009;94(2):662-669.

8. lacobellis G, Assael F, Ribaudo MC, et al. Epicardial fat from echocardiography: A new method for visceral adipose tissue prediction. Obes Res. 2003;11(2):304-310.

9. lacobellis G, Willens HJ. Echocardiographic epicardial fat: A review of research and clinical applications. J Am Soc Echocardiogr. 2009; 22(12):1311-1319; quiz 1417-1418.

10. Salami SS, Tucciarone M, Bess $R$, et al. Race and epicardial fat: The impact of anthropometric measurements, percent body fat and sex. Ethn Dis. 2013;23(3):281-285.

11. Gottdiener JS, Bednarz J, Devereux R, et al; American Society of Echocardiography. American Society of Echocardiography recommendations for use of echocardiography in clinical trials. J Am Soc Echocardiogr. 2004;17(10):1086-1119.

12. Lang RM, Bierig M, Devereux RB, et al; American Society of Echocardiography's Nomenclature and Standards Committee; Task Force on Chamber Quantification; American College of Cardiology Echocardiography Committee; American Heart Association; European Association of Echocardiography, European Society of Cardiology. Recommendations for chamber quantification. Eur J Echocardiogr. 2006;7(2):79-108.

13. Xing $Y, X u S$, Jia A, et al. Recommendations for revision of Chinese diagnostic criteria for metabolic syndrome: A nationwide study. J Diabetes. 2018;10(3):232-239.

14. Kim BJ, Kim BS, Kang JH. Echocardiographic epicardial fat thickness is associated with coronary artery calcification: Results from the CAESAR study. Circ J. 2015;79(4):818-824.

15. Calabuig A, Barba J, Guembe MJ, et al. Epicardial adipose tissue in the general middle-aged population and its association with metabolic syndrome. Rev Esp Cardiol (Engl Ed). 2017;70(4):254-260.

16. Fox CS, White CC, Lohman K, et al. Genome-wide association of pericardial fat identifies a unique locus for ectopic fat. PLoS Genet. 2012; 8(5):e1002705.

17. Silaghi A, Piercecchi-Marti MD, Grino M, et al. Epicardial adipose tissue extent: Relationship with age, body fat distribution, and coronaropathy. Obesity (Silver Spring). 2008;16(11):2424-2430.

18. Baragetti A, Pisano G, Bertelli $C$, et al. Subclinical atherosclerosis is associated with epicardial fat thickness and hepatic steatosis in the general population. Nutr Metab Cardiovasc Dis. 2016;26(2):141-153.

19. Abdallah E, El-Shishtawy S, Sherif N, Ali A, El-Bendary O. Assessment of the relationship between serum paraoxonase activity and epicardial adipose tissue in hemodialysis patients. Int Urol Nephrol. 2017; 49(2):329-335.

20. Kim BJ, Kang JG, Lee SH, et al. Relationship of echocardiographic epicardial fat thickness and epicardial fat volume by computed tomography with coronary artery calcification: Data from the CAESAR study. Arch Med Res. 2017;48(4):352-359. 